\title{
Xerostomia, Alteração do Paladar, da Saliva e da Sede: Percepção dos Idosos
}

\author{
Xerostomy, Alteration of Taste, Spittle and Thirst: Perception of Elderly \\ Clara Braga Pires ${ }^{1}$ \\ Gustavo Silva Costa ${ }^{2}$ \\ Isabela Santos Borges ${ }^{3}$ \\ Stéphanie Zuba Castro ${ }^{4}$ \\ Eliene de Oliveira ${ }^{5}$ \\ Maria Cleonice de Oliveira Nobre ${ }^{6}$ \\ Maria de Lourdes Carvalho Bonfim ${ }^{7}$
}

Resumo: Objetivos: Descrever e analisar a percepção dos idosos quanto à presença de xerostomia, alterações do paladar, da saliva e da sede. Metodologia: Trata-se de um estudo descritivo e transversal, com abordagem quantitativa em que utilizou-se o software IBM SPSS 22.0. Teve como população-alvo idosos, de ambos os sexos, capazes de responder às questões propostas, atendidos na Clínica Integrada III do curso de Odontologia da Universidade Estadual de Montes Claros e no Centro de Referência à Saúde do Idoso (CRASI) de Montes Claros-MG.

${ }^{1}$ Cirurgiã-Dentista (Unimontes). Residente em Saúde da Família (Universidade Estadual de Montes Claros) (Unimontes). Minas Gerais. Brasil. $₫$ clarinhabragapires@yahoo.com.br. (D) https://orcid.org/0000-0002-52602279

${ }^{2}$ Cirurgião-Dentista (Unimontes). Residente em Saúde da Família (Universidade Estadual de Montes Claros) (Unimontes). Minas Gerais. Brasil. \gustavocosta2905@gmail.com. (D https://orcid.org/0000-0003-0844-4610

${ }^{3}$ Cirurgiã-Dentista (Unimontes). Universidade Estadual de Montes Claros (Unimontes). Minas Gerais. Brasil. \isantosborges25@gmail.com. (D) https://orcid.org/0000-0002-9367-6397

${ }^{4}$ Cirurgiã-Dentista (Unimontes). Universidade Estadual de Montes Claros (Unimontes). Minas Gerais. Brasil. \zubacastro@gmail.com. (D) https://orcid.org/0000-0003-3542-835X

${ }_{5}^{5}$ Especialista em Saúde da Família (Unimontes). Universidade Estadual de Montes Claros (Unimontes). Minas Gerais. Brasil. \lyibl2007@hotmail.com. (D) https://orcid.org/0000-0001-9581-8574

${ }^{6}$ Especialista em Metodologia do Ensino Superior (Unimontes). Professora da Universidade Estadual de Montes Claros (Unimontes). Minas Gerais. Brasil. $₫$ cleonicenobre@yahoo.com.br. (D) https://orcid.org/0000-00019139-1278

${ }^{7}$ Doutora em Odontologia em Saúde Coletiva (UFMG). Professora da Universidade Estadual de Montes Claros (Unimontes). Minas Gerais. Brasil. $\triangle$ malu_cb2000@yahoo.com.br. (D https://orcid.org0000-000163994213

\begin{tabular}{c|ccc} 
Recebido em & Correções em & Aceito em & Publicado em \\
$12 / 04 / 2018$ & $31 / 10 / 2019$ & $07 / 09 / 2020$ & $14 / 12 / 2020$
\end{tabular}


A coleta de dados ocorreu no período de setembro a dezembro de 2015, pela aplicação de questionário semiestruturado, contendo questões abertas e fechadas, sob a forma de entrevista, que foi realizada após a aprovação pelo Comitê de Ética em Pesquisa da Universidade Estadual de Montes Claros sob Parecer n 50668915.1.0000.5146. Resultados: A maioria dos idosos era do sexo feminino (64\%); se encontrava na faixa etária acima de 70 anos (55\%); eram casados, divorciados ou viúvos (88\%); brancos (57\%); com escolaridade (93\%) e procedentes de outras localidades do norte do Estado de Minas Gerais (55\%). A maioria percebeu sensação de boca seca (79\%); que não há diminuição do paladar (58\%) e da sede $(51 \%)$, e que a saliva diminui (51\%). Conclusão: A compreensão das condições bucais dos pacientes idosos atendidos na Clínica Integrada III da Unimontes fez-se relevante, pois o conhecimento das percepções das alterações sentidas favoreceu a orientação dos cuidados com essa população.

Palavras-chave: Xerostomia. Sede. Saliva. Senescência. Saúde Bucal.

Abstract: Objectives: To describe and analyze the patients' perception regarding the presence of xerostomia, palate, saliva, and thirst alterations. Methodology: This is a descriptive and cross-sectional study with a quantitative approach using the IBM SPSS 22.0 software. The elderly population of both sexes was able to respond to the questions proposed at the Integrated Clinic III of the Dentistry course of the State University of Montes Claros and at the Center for Reference to Health of the Elderly (CRASI) of Montes Claros / MG. The data collection took place from September to December 2015, through the application of a semi-structured questionnaire, containing open and closed questions, in the form of an interview, which was performed after approval by the Research Ethics Committee of the State University of Montes Clear under opinion $\mathrm{n}^{\circ} .50668915 .1 .0000 .5146$. Results: Regarding gender, a predominance of female elderly (64\%) was observed. The prevalent age group was over 70 years $(55 \%)$; the majority of the elderly were married, divorced or widowed (88\%); whites (57\%); with education (93\%) and coming from other localities of the North of Minas (55\%). Most perceived dry mouth sensation (79\%). More than half (58\%) believe that there is no decrease in taste and thirst (51\%) and reports that saliva decreases $(51 \%)$. Conclusion: The understanding of the oral conditions of the elderly patients attending the Integrated Clinic III of Unimontes became relevant, since the knowledge of the perceptions of the changes felt by them favored the orientation of the care with this population.

Keywords: Xerostomy. Headquarters. Saliva. Senescence. Oral Health. 


\section{INTRODUÇÃO}

O envelhecimento da população tem sido um evento demográfico muito significativo desde o século passado que, de acordo com projeções populacionais da ONU, continuará nesse século, porém a intensidade do fenômeno será diferente com o passar dos anos (MONTENEGRO \& BRUNETTI, 2013). Entre os inúmeros fatores responsáveis pelo envelhecimento populacional, destaca-se o aumento da expectativa de vida, principalmente nos países em desenvolvimento, como é o caso do Brasil.

O aumento da expectativa de vida nunca atingiu números tão altos, devido, principalmente, a implementação de políticas públicas de saúde de medicina preventiva resultantes dos avanços da pesquisa científica (CARDOSO; LAGO, 2010).

As mudanças na estrutura etária da população acarretam doenças próprias do envelhecimento. Essas doenças ganham maior expressão à medida que a sociedade envelhece e desafiam os sistemas de saúde (KALACHE, 2008; VERAS, 2009).

Como a saúde bucal não está desvinculada da saúde geral, é importante salientar que a cavidade oral também sofre muitas alterações, como xerostomia; hipossalivação; alterações gustativas; doença periodontal; modificação na percepção de sede; cárie radicular; e outras, que podem ser próprias do envelhecimento ou resultar do uso de vários medicamentos. Cabe ao cirurgião-dentista detectá-las e saber como intervir, propiciando melhor qualidade de vida aos idosos (CARDOSO; LAGO, 2010; FREITAS, LOCK, UNFER, 2013). Neste estudo foram abordadas as seguintes alterações bucais: xerostomia, alterações do paladar, da saliva e da sede.

A xerostomia é uma condição de sensação subjetiva de boca seca que pode ou não estar associada à hipossalivação; manifestação de algumas doenças sistêmicas, tais como a Síndrome de Sjögren e a Síndrome da Imunodeficiência Adquirida (AIDS); tratamento radioterápico; tabagismo e uso de certos fármacos (FERREIRA et al., 2010; CÔRTE-REAL, FIGUEIRAL, CAMPOS, 2011; RECH, 2016).

Já as alterações gustativas nos idosos são consequências inerentes à senescência, visto que elas são provocadas por uma diminuição acentuada do número de botões gustativos, responsáveis pela percepção dos gostos primários: salgado, doce, ácido, amargo e umami. Um indivíduo jovem possui cerca de 250 botões gustativos por papila, em contrapartida, após os 70 anos de idade esse número cai para 100 (PAULA et al., 2008; DA SILVA et al., 2015; PASSOS, GUIMARÃES, VICTORIA, 2016). 
A saliva é um componente de fundamental importância para a cavidade oral visto que participa de processos como desmineralização e remineralização, produção de amilase salivar, que inicia o processo digestivo, retenção de próteses e tamponamento. No processo de envelhecimento há uma diminuição na quantidade e na qualidade da saliva, uma vez que ela se torna mais viscosa devido a alterações provocadas por uma degeneração das glândulas salivares (DA i; SÁLVIO; MIRANDA, 2012; FERNANDES-COSTA et al., 2013).

Também há uma correlação dessas alterações com o uso de medicamentos. No mercado existem cerca de 250 fármacos que provocam esse efeito adverso, e idosos são mais susceptíveis por serem pacientes muitas vezes polifármacos (LOPES et al., 2015). Um grande problema dessa situação é que, muitas vezes, para compensar a diminuição do paladar, os idosos consomem uma dieta rica em açúcar e sal, o que pode acarretar ou acentuar doenças como diabetes mellitus e hipertensão arterial (PAULA et al., 2008; LOPES et al., 2015).

Além disso, é válido mencionar que a substância mais importante para a manutenção da vida é a água, que nos idosos deve corresponder a 40-50\% do peso corporal, visto que desempenha funções indispensáveis para a manutenção da saúde. Devido às alterações decorrentes da senescência, o consumo diário de água nem sempre equivale ao necessário, em virtude de uma disfunção cerebral ou de uma diminuição da sensibilidade dos osmorreceptores, podendo ter como consequências: desidratação, cefaleia, insuficiência renal, confusão, delírio e infecções urinárias (MENDONÇA et al. 2015; GENARO, GOMES, IENAGA, 2016).

Neste contexto, esse estudo se justifica pela relevância do conhecimento do cirurgiãodentista acerca da percepção da saúde bucal dos idosos, posto que esse público passa por diversas e constantes alterações decorrentes do envelhecimento. Também o rápido envelhecimento populacional exige que os profissionais de saúde conheçam a realidade das populações onde atuam, o que possibilitará a definição dos problemas de saúde prioritários e o planejamento de ações mais efetivas para a solução desses problemas.

Diante do que foi exposto, objetivou-se, por meio deste estudo, descrever e analisar a percepção dos idosos quanto à presença de xerostomia, alterações do paladar, da saliva e da sede.

\section{METODOLOGIA}

Trata-se de um estudo descritivo transversal, com abordagem quantitativa, que teve como população-alvo idosos (pessoas com 60 anos ou mais de idade), de ambos os sexos, com 
capacidade de responder às questões propostas, atendidos na Clínica Integrada III do curso de Odontologia da Universidade Estadual de Montes Claros e no Centro de Referência à Saúde do Idoso (CRASI) de Montes Claros/MG. Constituiu critério de exclusão: idosos que apresentaram dificuldades cognitivas, de compreensão, de raciocínio e/ou de comunicação, para responder o questionário.

A coleta de dados ocorreu no período de setembro a dezembro de 2015, pela aplicação de questionário semiestruturado, contendo questões abertas e fechadas, sob a forma de entrevista, que foi realizada após a aprovação pelo Comitê de Ética em Pesquisa da Universidade Estadual de Montes Claros sob o Parecer nº 50668915.1.0000.5146.

Participaram do estudo 100 idosos que estavam em tratamento odontológico na Clínica Integrada III e atingiram o critério de elegibilidade.

O questionário continha seis grupos de grandes variáveis. Como este estudo é um recorte de uma pesquisa maior, foram trabalhados somente dois grupos com as seguintes variáveis: perfil dos idosos da clínica Integrada III (idade, sexo, estado civil, cor, renda, escolaridade e procedência); alterações bucais percebidas (sensação de boca seca-xerostomia-, diminuição do paladar, diminuição da saliva e diminuição da sede).

Após a coleta de dados, efetuou-se a análise estatística descritiva utilizando-se o software IBM SPSS 22.0. A descrição dos resultados foi expressa em tabelas de frequência. Foram associadas às variáveis independentes (perfil dos idosos) com as variáveis resposta (alterações bucais percebidas). Essa associação foi testada pelo quiquadrado e adotou-se um nível de significância de $\mathrm{p}<0,05$.

\section{RESULTADOS}

Observou-se uma predominância de idosos do sexo feminino (64\%), que se encontravam na faixa etária acima de 70 anos (55\%); eram casados, divorciados ou viúvos (88\%); de cor branca (57\%); possuíam escolaridade (93\%) e eram procedentes de outras localidades do norte do Estado de Minas Gerais (55\%) (TABELA 1).

A Tabela 2 descreve as alterações bucais percebidas pelos idosos. A maioria percebeu sensação de boca seca (79\%); que não há diminuição no paladar (58\%) e da sede (51\%) e que a saliva diminui $(51 \%)$.

A Tabela 3 evidencia a associação das variáveis de percepção com o perfil dos idosos. Verificou-se que parte dos entrevistados (41\%) com idade entre 60 e 70 anos relataram ter a 
sensação de boca seca, assim como os que se encontram em uma faixa etária acima de 70 anos, p=0,025. Em relação às alterações do paladar, os idosos de 60 a 70 anos, em sua maioria (26\%) relataram essa alteração, ao contrário do que se observa entre os idosos com idade superior a 70 anos, que não perceberam modificações gustativas (39\%), p=0,00. Quanto a percepção da alteração da saliva, os idosos de 60 a 70 (31\%) relatam diminuição desta variável, enquanto os idosos acima de 70 anos (35\%) relataram não perceber tais modificações, p=0,004.

A Tabela 4 descreve a relação encontrada entre as variáveis paladar e saliva com o estado civil dos participantes, em que 7\% dos solteiros (maioria) relataram que há alteração do paladar, enquanto que $53 \%$ dos casados/divorciados/separados/viúvos, também correspondente a maioria, relataram a não percepção desta alteração, $p=0,000$. Quanto a percepção da diminuição da saliva, 7\% dos solteiros (maior parte), relataram não as apresentar, ao passo que $46 \%$ dos casados/divorciados/separados/viúvos (maioria) mencionaram ter percebido alterações no paladar, $\mathrm{p}=0,004$.

\section{DISCUSSÃO}

A percepção da saúde bucal dos idosos é uma variável que demonstra a experiência subjetiva dos mesmos sobre seu próprio bem estar funcional, social e psicológico. Alguns fatores são associados a essa auto-avaliação, podendo ser referentes ao ambiente externo e ao indivíduo (MARTINS; BARRETO; PORDEUS, 2009).

O ambiente externo diz respeito ao local de residência e ao sistema de saúde disponível e no nível individual, essa auto-avaliação é influenciada por fatores relacionados à saúde, tais como: idade; sexo; raça e fatores de predisposição, como escolaridade e alfabetização em saúde para cuidados preventivos. Renda pessoal e familiar também são recursos que se enquadram no nível individual, bem como a adesão a um plano de saúde (MARTINS; BARRETO; PORDEUS, 2009).

No atual estudo, os fatores relacionados ao indivíduo e ao ambiente externo foram levados em consideração, incluindo, o estado civil que evidenciou idosos casados, divorciados ou viúvos, na sua maior parte. Da Luz et al. (2014), em pesquisa quantitativa, descritiva e transversal, com idosos residentes em um município da região norte do Rio Grande do Sul e que objetivou descrever o perfil epidemiológico da população, observaram que em relação à situação conjugal dos idosos, $53,8 \%$ eram casados e $32,1 \%$ viúvos, o que ratifica os dados sobre estado civil encontrados no presente estudo. 
Em relação ao sexo, a maioria dos entrevistados da Clínica Integrada III da Unimontes, participantes da pesquisa, eram do sexo feminino (64\%). Essa predominância de mulheres vai ao encontro com o censo do IBGE de 2010, em que a população idosa brasileira era composta por 25,4 milhões de indivíduos com 16,9 milhões (56\% dos idosos) do sexo feminino e 13,3 milhões (44\% dos idosos) do sexo masculino. Cardoso; Lago (2010) realizaram uma pesquisa do tipo transversal com abordagem quantitativa em um Centro de Convivência da Terceira Idade (CCTI), em que dos 80 participantes a maioria também era composta por mulheres, o que corrobora com os dados encontrados neste estudo.

Freitas; Lock; Unfer (2013) realizaram uma pesquisa com pessoas de idade igual ou superior a 60 anos no Hospital Universitário de Santa Maria (RS), no ano 2012. Os achados evidenciaram que mais da metade dos idosos (59\%) apresentavam queixa de boca seca durante grande parte do dia, o que corrobora com os dados encontrados neste estudo, visto que $79 \%$ dos idosos da Clínica Integrada III da Unimontes também relataram essa sensação. Côrte-Real; Figueiral; Campos (2011) justificam esses resultados pelos efeitos xerostomizantes de mais de $80 \%$ dos fármacos utilizados pelos indivíduos dessa faixa etária, como os sedativos; antipsicóticos; anti-depressivos; anti-hipertensivos; anti-histamínicos e citotóxicos.

Nesta pesquisa, observou-se que a maioria dos idosos (51\%) teve a autopercepção da diminuição quantitativa do fluxo salivar. Todavia, tal resultado vai em desencontro com o que Ferreira et al. (2010) obtiveram em uma pesquisa, na qual 46\% da amostra (composta por 98 idosos, com idade entre 60 e 86 anos) relataram não apresentar essa redução. Entretanto, Silva; Bonini; Bringel (2015) em estudo com 38 idosos de 65 a 101 anos de dois asilos do município de Araguaína/TO, verificaram que 19 idosos (50\%) mencionaram ter hipossalivação. Esse processo que acontece no processo de envelhecimento, é provocado por uma degeneração gradativa das glândulas salivares que desencadeia a hipofunção das mesmas, o que leva a uma diminuição quantitativa e alteração qualitativa (ser mais viscosa) da saliva, principalmente quando o idoso encontra-se em repouso (ROSA et al., 2008; CÔRTE-REAL, FIGUEIRAL, CAMPOS, 2011)

Em relação à diminuição do paladar, 58\% dos entrevistados da Clínica Integrada III da Unimontes, mencionaram não apresentar tal alteração, principalmente quando associado a idade. Os idosos acima de 70 anos (39\%) não percebem essa alteração. Esses dados são corroborados pela literatura analisada, considerando que Passos; Guimarães; Victoria (2016) em pesquisa composta por uma amostra de 40 indivíduos, na qual 20 eram adultos com idade entre 18 a 25 anos e 20 eram idosos com idade entre 60 a 85 anos de ambos os sexos, observaram 
que a maioria dos idosos necessitavam de uma quantidade maior de açúcar e sal para que pudessem perceber os sabores dos alimentos. Segundo Rosa et al. (2008) 80\% dos indivíduos acima dos cinquenta anos apresentam redução na capacidade gustativa. Essas alterações são provocadas por uma diminuição gradual da quantidade de botões gustativos por papila principalmente após os 70 anos, o que leva a uma redução do limiar de detecção e reconhecimento dos sabores primários (amargo, doce, ácido, salgado e umami) (ROSA et al., 2008; LOPES et al., 2015; PASSOS, GUIMARÃES, VICTORIA, 2016).

É válido mencionar também que, com o envelhecimento, a homeostasia diminui, de forma que os idosos sintam menos sede e, consequentemente, tomem uma menor quantidade de líquidos. Estudo realizado em Presidente Prudente, junto a 85 idosos, constatou que 55,55\% tomam água o dia todo, contudo, a quantidade de água ingerida não é a ideal para que ela possa exercer suas funções (GENARO; GOMES; IENAGA, 2016). Esse dado é preocupante, haja vista que a água é um nutriente fundamental para a saúde dos indivíduos, pois regula a temperatura corporal; participa dos processos digestivo, respiratório, cardiovascular e renal; do transporte de nutrientes e da eliminação de substâncias tóxicas (CARVALHO; ZANARDO, 2010). No presente estudo, a maioria dos idosos pesquisados (51\%) relatou diminuição na sensação de sede, em acordo com estudos de Carvalho; Zanardo (2010) e Genaro; Gomes; Ienaga (2016), que também encontraram redução da percepção de sede.

Neste estudo evidenciou-se a associação das variáveis, boca seca, paladar e saliva com o perfil dos idosos. Em relação à sensação de boca seca, tanto os idosos de 60 a 70 ou mais de 70 anos, relataram a percepção dessa sensação (TABELA 3).

Em relação à modificação do paladar, os indivíduos pertencentes à faixa etária de 60 a 70 anos, em sua maior parte (26\%), mencionaram alteração da sensação gustativa, ao passo que houve prevalência de resposta negativa (39\%) ao se avaliar indivíduos com idade superior a 70 anos no presente estudo. Contudo, Paula et al. (2008) reiteram que essas alterações tendem a se iniciar por volta dos 70 anos ou mais, estando relacionadas à redução da sensibilidade pelos gostos primários. Dessa forma, não só a habilidade de perceber as alterações gustativas fica comprometida com o avançar da idade, mas também a de discernir os sabores, tendo origem multicausal e ocorre, principalmente, em decorrência de uma redução do tamanho e da quantidade das papilas gustativas. Além disso, modificações da cavidade bucal provocadas por doenças, problemas dentários e medicações provocam uma diminuição fisiológica da sensibilidade aos sabores. Essa diferença entre os resultados pode ter relação com o fato de ser uma alteração com caráter puramente subjetivo (PAULA et al., 2008). 
Ao analisar a diminuição do fluxo salivar associada à idade dos entrevistados, notou-se menor percepção dessa alteração pelos idosos acima de 70 anos (35\%) em comparação com os de idade entre 60 a 70 anos (14\%). Essa constatação está em desacordo com estudo, no qual Silva; Bonini; Bringel (2015) esclarecem que quanto mais idoso o paciente, maior a tendência de redução do fluxo salivar.

Pode-se também observar neste estudo que houve uma associação entre as variáveis paladar e saliva com o estado civil dos entrevistados $(\mathrm{p}<0,05)$. Entre os solteiros há maior percepção de alteração do paladar do que entre os casados/divorciados/separados/viúvos, 35\% mencionaram presença da alteração e 53\% relataram não a possuir. No entanto, oscasados/divorciados/separados/viúvos perceberam diminuição da saliva mais do que os solteiros.

Lopes et al. (2015) avaliaram 203 idosos com idade variando de 60 a 98 anos. Desses, $75 \%$ eram casados, $14,3 \%$ viúvos, $6,4 \%$ divorciados, $3,4 \%$ solteiros e $1 \%$ referiu ter relação estável e todos apresentaram alterações gustativas, justificadas pelo uso crônico de medicamentos utilizados pela população estudada, modificando principalmente o sabor doce. Esse evento provavelmente explica a pouca percepção dos idosos à mudança do paladar pois os idosos entrevistados, casados/divorciados/separados/viúvos são maioria e têm mais de 70 anos. Além do mais, o resultado desta pesquisa, opoente à literatura consultada, pode ser explicado pelo fato de existir naturalmente uma diferença entre as pessoas, em relação à percepção e tolerância dos sabores, como, no sabor doce que é mais perceptível para quem apresenta maior número de papilas fungiformes (PAULA et al., 2008).

\section{CONCLUSÃO}

O envelhecimento populacional é um evento real, irreversível e universal, e que ocasiona muitas alterações fisiológicas no corpo humano, e são observadas, também, na cavidade bucal.

Nesta pesquisa, identificou-se que os idosos entrevistados tinham mais de 70 anos; eram do sexo feminino; apresentaram sensação de boca seca; perceberam que a saliva diminui; e que não houve diminuição do paladar e da sede.

A sensação de boca seca se configura como uma alteração predominante, independentemente da faixa etária dos entrevistados. Contudo, o analisar as variações referentes ao paladar e a saliva notou-se que os idosos de 60 a 70 anos relataram a percepção de alterações 
dessas variáveis, ao contrário do que se vê entre os idosos com idade superior a 70 anos. Para que esses dados sejam confirmados há necessidade de pesquisas com delineamento mais elaborado.

Mesmo assim, verificou-se que a compreensão das condições bucais dos pacientes idosos atendidos na Clínica Integrada III da Unimontes fez-se relevante, pois o conhecimento das percepções das alterações sentidas nessa amostra favoreceu a orientação dos cuidados a essa população.

\section{REFERÊNCIAS}

1 MONTENEGRO, Fernando Luiz Brunetti.; MARCHINI, Leonardo. Odontogeriatria: Uma visão gerontológica. Rio de Janeiro. Editora Elsevier, 2013.

2 CARDOSO, Maria Beatriz Ribeiro.; LAGO, Eliana Campêlo. Alterações bucais em idosos de um centro de convivência. Revista Paraense de Medicina, v. 24, n. 2, p. 35-41, 2010.

3 KALACHE, Alexandre. O mundo envelhece: é imperativo criar um pacto de solidariedade social. Ciência \& Saúde Coletiva, v. 13, p. 1107-1111, 2008

4 VERAS, Renato. Envelhecimento populacional contemporâneo: demandas, desafios e inovações. Revista de Saúde Pública, v. 43, p. 548-554, 2009.

5 FREITAS, Daniela Napoleão.; LOCK, Nicássia Cioquetta.; UNFER, Beatriz. Hipofunção das glândulas salivares em idosos hospitalizados relacionada a medicamentos. Geriatrics, Gerontology and Aging, v. 7, n. 3, p. 179-183, 2013.

6 FERREIRA, Olívia Galvão Lucena et al. O envelhecimento ativo sob o olhar de idosos funcionalmente independentes. Revista da Escola de Enfermagem da USP, v. 44, n. 4, p. $1065-$ $1069,2010$.

7 CÔRTE-REAL, Inês S.; FIGUEIRAL, Maria Helena.; CAMPOS, José Carlos Reis. As doenças orais no idoso-Considerações gerais. Revista Portuguesa de Estomatologia, Medicina Dentária e CirugiaMaxilofacial, v. 52, n. 3, p. 175-180, 2011. 
8 RECH, Carlos Alberto.; MEDEIROS, Aline Wilke. Xerostomia associada ao uso de medicamentos em idosos. Journalof Oral Investigations, v. 5, n. 1, p. 13-18, 2016.

9 PAULA, Roberta da Silva et al. Alterações gustativas no envelhecimento. Revista Kairós, p. 217-235, 2008.

10 DA SILVA, T. Met al. Alteração no paladar de idosos e suas repercussões nutricionais. Anai's CIEH, vol. 2, N.1, 2015.

11 PASSOS, Jussara Gonçalves.; GUIMARÃES, Lucas Costa.; VICTORIA, Maria do Carmo Malicheski. Avaliação da percepção gustativa em idosos para os gostos básicos, doce e salgado, em comparação a jovens adultos. Journal of the Health Sciences Institute, v. 34, n. 1, p. 29-32, 2016.

12 DA SILVA, Raíssa Gabriela Nogueira.; SALVIO, Luisa Amaral.; MIRANDA, Alexandre Franco. Alterações bucais na terceira idade: uma realidade clínica do futuro cirurgião-dentistabreves considerações. Revista Portal de Divulgação, n. 24, 2012.

13 FERNANDES-COSTA, Anderson Nicolly et al. As Principais Modificações Orais que ocorrem durante o Envelhecimento. Revista Brasileira de Ciências da Saúde, v. 17, n. 3, p. 293-300, 2013.

14 LOPES, Ana Carolina Freire et al. Prevalência de alterações gustativas em idosos em uso crônico de fármacos. Geriatrics, Gerontology and Aging, v. 9, n. 4, p. 132-137, 2015.

15 MENDONÇA, L. F et al. Influência da água na desidratação de idosos. IV Congresso Internacional de Envelhecimento Humano. 2015.

16 GENARO, Sandra.; GOMES, Fernanda. Helena. Marques; IENAGA, Kátia Kaori. Análise do consumo de água em uma população de idosos. In: Colloquium Vitae. ISSN: 1984-6436. 2016. p. 01-12.

17 MARTINS, Andréa Maria Eleutério de Barros Lima.; SANDHI, Maria Barreto.; PORDEUS, Isabela Almeida.Auto-avaliação de saúde bucal em idosos: análise com base em modelo multidimensional. Cadernos de Saúde Pública, v. 25, p. 421-435, 2009.

18 Instituto Brasileiro de Geografia e Estatística (IBGE) Censo Demográfico 2010. 
19 DA LUZ, Elizangela Plestchet al. Perfil sociodemográfico e de hábitos de vida da população idosa de um município da região norte do Rio Grande do Sul, Brasil. Revista Brasileira de Geriatria e Gerontologia, v. 17, n. 2, p. 303-314, 2014.

20 SILVA, Briza Letícia Almeida.; BONINI, Julia Acker.; BRINGEL, Fabiana de Andrade. Condição de saúde bucal de idosos institucionalizados em Araguaína/TO. Braz J Periodontol, v. 25, n. 1, p. $7-13,2015$.

21 ROSA, Lâner et al. Odontogeriatria- A saúde bucal na terceira idade. Revista da Faculdade de Odontologia-UPF, v. 13, n. 2, 2008.

22 CARVALHO, Ana Paula Lambrecht.; ZANARDO, Vivian Polachini Skzypek. Consumo de água e outros líquidos em adultos e idosos residentes no município de Erechim-Rio Grande do Sul. Revista Perspectiva, Erechim, v. 34, n. 125, p. 117-126, 2010. 
Tabela 1. Perfil dos idosos da Clínica Integrada III/Unimontes. Montes Claros/MG, 2018

\section{Variáveis}

Idade

Sexo

$$
\text { Feminino }
$$

Masculino

Total

Estado Civil Solteiro

Casado/Divorciado/Viúvo

Total

Cor

Branco

Não branco

\section{Frequência}

45

55

100

64

36

100

\section{2}

88

100

\section{7}

43

\begin{tabular}{llcc} 
Renda & Não possui renda & 5 & 5 \\
& Possui renda & 95 & 95 \\
Escolaridade & Sem escolaridade & 7 & 7 \\
& Com escolaridade & 93 & 93 \\
\multirow{2}{*}{ Procedência } & Montes Claros-MG & 45 & 45 \\
& Outros & 55 & 55 \\
\hline
\end{tabular}

Fonte: Clínica Integrada III.
57

43

64

36

12

88

3


Tabela 2. Xerostomia, alterações do paladar, da saliva e da sede percebidas pelos idosos da Clínica de Integrada III/Unimontes. Montes Claros/MG, 2018

\begin{tabular}{|c|c|c|c|}
\hline Variáveis & & Frequência & Porcentagem \\
\hline $\begin{array}{l}\text { Sensação de boca seca } \\
\text { (Xerostomia) }\end{array}$ & $\begin{array}{l}\text { Sim } \\
\text { Não }\end{array}$ & 21 & $\begin{array}{l}79 \\
21\end{array}$ \\
\hline Diminuição do paladar & $\begin{array}{l}\text { Sim } \\
\text { Não }\end{array}$ & $\begin{array}{l}42 \\
58\end{array}$ & $\begin{array}{l}42 \\
58\end{array}$ \\
\hline Diminuição da saliva & $\begin{array}{l}\text { Sim } \\
\text { Não }\end{array}$ & $\begin{array}{l}51 \\
49\end{array}$ & $\begin{array}{l}51 \\
49\end{array}$ \\
\hline Diminuição da sede & $\begin{array}{l}\text { Sim } \\
\text { Não }\end{array}$ & $\begin{array}{l}47 \\
53\end{array}$ & $\begin{array}{l}47 \\
53\end{array}$ \\
\hline
\end{tabular}

Fonte: Clínica Integrada III.

TABELA 3. Associação das variáveis de percepção - boca seca, paladar, saliva- com idade dos idosos, 2018

\begin{tabular}{cccccccc}
\hline Variável & 60 a 70 anos & TOTAL & $\begin{array}{c}\text { Acima de 70 } \\
\text { anos }\end{array}$ & TOTAL & P VALOR \\
& SIM & NÃO & \multicolumn{5}{c}{$\begin{array}{c}\text { SIM } \\
\text { NÃO }\end{array}$} \\
\hline Boca Seca & 41 & 4 & 45 & 38 & 17 & 55 & 0,025 \\
Paladar & 26 & 19 & 45 & 16 & 39 & 55 & 0,00 \\
Saliva & 31 & 14 & 45 & 20 & 35 & 55 & 0,004 \\
\hline
\end{tabular}

Fonte: Clínica Integrada III. 
Tabela 4. Associação das variáveis de percepção -paladar e saliva - com estado civil dos idosos, 2018

\begin{tabular}{|c|c|c|c|c|c|c|c|}
\hline \multirow[t]{2}{*}{ Estado Civil } & \multicolumn{2}{|c|}{ Solteiro } & TOTAL & \multicolumn{2}{|c|}{ Viúvo } & \multirow[t]{2}{*}{ TOTAL } & \multirow[t]{2}{*}{ P VALOR } \\
\hline & \multicolumn{2}{|l|}{$\begin{array}{l}\text { SIM } \\
\text { NÃO }\end{array}$} & & SIM & NÃO & & \\
\hline Paladar & 7 & 5 & 12 & 35 & 53 & 88 & 0,000 \\
\hline Saliva & 5 & 7 & 12 & 46 & 42 & 88 & 0,004 \\
\hline
\end{tabular}

Fonte: Clínica Integrada III. 